\title{
Productive faculty resources activated by curricular materials: An example of epistemological beliefs in University Modeling Instruction
}

\author{
Daryl McPadden $\odot$ \\ Department of Physics and Astronomy, Michigan State University, \\ 567 Wilson Road, East Lansing, Michigan 48824, USA \\ Eric Brewe \\ Physics Department and School of Education, Drexel University, \\ 3141 Chestnut Street, Philadelphia, Pennsylvania 19104, USA \\ STEM Transformation Institute, Department of Teaching and Learning, and Department of Physics, \\ Florida International University, 11200 SW 8th Street, Miami, Florida 33199, USA \\ Camila Monsalve $\odot$ \\ Department of Physics and Astronomy, Michigan State University, \\ 567 Wilson Road, East Lansing, Michigan 48824, USA \\ Department of Physics and STEM Transformation Institute, Florida International University, \\ 11200 SW 8th Street, Miami, Florida 33199, USA \\ Vashti Sawtelle® \\ Lyman Briggs College and Department of Physics \& Astronomy, Michigan State University, \\ 567 Wilson Rd, East Lansing, Michigan 48824, USA
}

(Received 9 July 2019; accepted 24 December 2019; published 4 December 2020)

\begin{abstract}
[This paper is part of the Focused Collection on Curriculum Development: Theory into Design.] When disseminating curricula, typically only the physical curriculum materials are transferred from the instructor to another. However, left as artifacts in the curricula are a set of epistemological beliefs held by the creators and designers of the materials. These epistemological beliefs are often underpinning the activity structures and strongly influence how the curriculum materials are implemented in the classroom, particularly when those beliefs differ from those activated by the instructor. In this paper, we articulate three epistemological beliefs that are built into the University Modeling Instruction curriculum that are essential for running the large-group discussions, which are a crucial part of the curriculum. We highlight how these beliefs are built into the student-facing and instructor-facing curricular materials and the influence of these beliefs in the interpretation and implementation of the curriculum materials. Finally, we discuss the implications on curriculum dissemination, on the adoption and adaptation of curriculum materials, and training models for instructors beyond the curriculum designers.
\end{abstract}

DOI: 10.1103/PhysRevPhysEducRes.16.020158

\section{INTRODUCTION}

Over the past several decades, science educators and education researchers have developed a variety of active learning curricula. In physics alone, these curricula have taken a wide variety of forms from the addition of

Published by the American Physical Society under the terms of the Creative Commons Attribution 4.0 International license. Further distribution of this work must maintain attribution to the author(s) and the published article's title, journal citation, and DOI. clicker questions to introductory physics lectures to integrating laboratory activities and student discussions [1-8]. Frequently, these curricula follow a pattern of development: materials are created (including studentfacing and instructor-facing documents) typically at a single institution, there is a publication introducing the curriculum and its structures, evaluative measures are assessed, materials are revised, and the curriculum is shared with others via workshops, dissemination of materials, or shared by word of mouth. Despite the demonstrated benefits of active learning $[9,10]$, adoption of active learning curricula is still slow to occur, often with pushback from faculty, departments, and institutions [11,12]. 
While there is likely a combination of reasons that is unique to each context, one common barrier to the adoption of active learning curricula could be a mismatch between the beliefs about knowing and learning assumed by curriculum designers and the epistemological resources that same curriculum activates for instructors [11]. For example, an instructor who views teaching as transmitting information is less likely to find more dramatic reforms of teaching useful, but may experiment with reforms like Peer Instruction or flipped classrooms. These are both reforms which preserve many of the information transfer elements of teaching [13]. Similarly, faculty with a strong experimental bent may view learning as happening through experimentation and thus may find curricula which prioritize the role of experimentation attractive, such as the Investigative Science Learning Environment (ISLE) [5].

Particularly in active learning curricula, developers are often purposefully challenging student and instructor beliefs, including: (1) what it means to "do physics" in the classroom (e.g., running experiments or solving problems rather than taking notes from lecture), (2) what it means "teach physics" (e.g., facilitating discussion or asking questions rather than telling), and (3) what it means to "learn physics" (e.g., working in small groups rather than being a solitary learner). Curriculum designers attempt to build cues about these epistemological beliefs into the curricular materials. However, in the process of sharing curriculum materials with instructors, these beliefs are rarely stated; rather curriculum developers often assume the curriculum will cue similar epistemological resources in the instructors preparing to use the materials. Especially in the curricula where the epistemological beliefs of curriculum designers are significantly different from those held in a traditional lecture course, articulating these underpinnings to the materials is crucial to being able to adopt and adapt the curriculum. In this paper, we will use an example from our own development experience to demonstrate how these curricular materials represent artifacts of the designers' beliefs that can serve to prime and cue productive instructor epistemological resources. We will examine materials from the University Modeling Instruction (UMI) curriculum, which is built from significantly different beliefs about the nature of knowing and learning than those assumed in a lecture classroom.

\section{A. University Modeling Instruction}

University Modeling Instruction is a calculus-based, introductory physics curriculum, which is centered around students constructing conceptual physics models in a studio-style integrated laboratory and lecture environment. The curricular materials for UMI have been developed assuming a two-semester course sequence, with the first semester covering introductory mechanics topics and the second covering introductory electricity and magnetism. The activities in UMI follow a modeling cycle [14-16], starting with the investigation of the phenomenon of interest, coordinating representations through abstraction and generalization of the model, and finally the application and refinement of the model. The purpose of this modeling cycle is to scaffold students through the process of creating, testing, applying, and refining a physical model, which simulates the process that scientists follow in their work [17-21].

Each activity within the modeling cycle is then structured to follow a student participation cycle [21]. Students start the activities by working in small groups (3-6 students). As they complete the activities, they are typically asked to answer the following three questions on small whiteboard. (1) What did you learn? (2) What rules can you make or what patterns to did you see? (3) What is still unclear or what do you still have questions about? After students have created their whiteboards, they come together for a large group discussion consisting of 4-8 groups ( $\sim 30-40$ students). The large group discussions are often referred to as a "board meeting."

The purpose of the board meeting is for students to be able to share what they learned, what they found, and to be able to come to consensus about the conceptual ideas in each activity. Depending on the type of activity and the position of the activity in the modeling cycle, the goal of the board meeting may be slightly different. For example, in an initial investigation activity, the goal of the board meeting may be to elicit questions about the phenomenon and to share the results of empirical testing. Or for a coordination activity, the goal may be to create a list of rules for how to use a particular representation, like a free-body diagram. In any board meeting, an underlying assumption is that students will take the lead of the discussion. Ideally, students are expected to share ideas, critique each others' work, and ask and answer each others' questions. At the end of the board meeting, students then return to their small groups to continue on to the next activity, thus starting the cycle over again. These two cycles-the modeling cycle and the student participation cycle-distinguish UMI from other studio-style curricula that have been developed.

Underpinning the UMI courses is a set of intersecting theories: the Modeling Theory of Science (MTS) and the Modeling Theory of Instruction (MTI). As described by Brewe and Sawtelle, the modeling theory of science states that modeling is the central practice of science [21]. The Modeling Theory of Instruction then outlines how students can productively participate in the creation of scientific models in a classroom environment [16,22]. The application of the MTS and MTI in the classroom has already been described $[21,23]$. From an evaluation standpoint, the UMI curriculum has been shown to be successful across multiple measures such as conceptual gains, drop-fail-withdraw rates, attitudinal measures, use of representational tools, and student participation in class [24-29]. 
While the successes and theoretical backing have been well documented for UMI, the role of the instructor in these courses has been underemphasized. Desbien [30], and later expanded on by Durden et al. [31], described a set of instructional strategies and heuristics (known as Modeling Discourse Management), which are meant to help instructors facilitate the discourse in a UMI classroom; however, Modeling Discourse Management does not focus on leading the board meeting discussions. The board meeting itself does not have student-facing materials that guide the discussion but is instead the culmination of the classroom's work in UMI during each student participation cycle. Thus, the board meeting is the primary place where the underpinning epistemological beliefs of the UMI curricula designers (as well as the missing support structure for new instructors) become apparent.

With this paper, we aim to articulate the epistemological resources that UMI designers intended to cue in the UMI curricula, with a particular focus on the aspects related to leading the board meeting. We examine the curriculum as an artifact of the beliefs of the curriculum designers and ask which artifacts are most likely to successfully cue the intended resources for classroom instructors and which are likely to cue unintended resources. We will focus on our discussion primarily on the role of the "lead instructor" in the course; however, we recognize that much of the discussion is also applicable to supporting instructors, including graduate teaching assistants and undergraduate learning assistants. In the following sections, we will outline the epistemological beliefs that are intended to be communicated by the designers of UMI, introduce a set of UMI studentfacing and instructor-facing materials, and outline how those materials exemplify successful curing of similar epistemological resources. Then we will describe how the cued resources impact the interpretation of the curricular materials, which is crucial for running a board meeting in UMI. Finally, we will close with a discussion of how curricula designers could better support instructors in understanding the epistemological beliefs built into their materials.

\section{THEORY}

In this section, we describe our orientation to epistemological resources, beliefs, and framing. We intend to align our work with previous physics education researchers, while also acknowledging that this theory has a much longer and richer history [32-34]. We then describe the specific epistemological beliefs held by the curriculum designers of UMI. We outline these beliefs as a precursor for examining cues for similar resources in the curricular materials.

\section{A. Our orientation to epistemological resources, beliefs, and framing}

Framing a classroom is a continuous process of making interpretations about how to act in a particular situation.
Framing answers the question, "What is it that is happening here?" [33]. Epistemological framing is the dynamic process of answering the question, "What forms of knowing and learning are appropriate here?" [35]. Answering this question is a dynamic process where one's prior knowledge about a setting interacts with the physical and social cues of the environment. In the classroom, Hammer and Elby have argued that an individual's prior knowledge is composed of epistemological resourcesfine-grained ideas about the nature of knowing and learning $[36,37]$.

Traditionally, when physics education researchers discuss epistemological framing their focus is on how students frame the classroom and the epistemological resources that students activate in constructing that framing [38-40]. Recently the emphasis has been on understanding how to support productive epistemological orientations toward science [38,39]. Specifically, Redish and Hammer describe how they designed a study to understand "the match or mismatch between student epistemologies and what we want them to learn..." [38]. Redish and Hammer describe the learning of these epistemologies as the implicit curriculum.

We argue that designers of curricular materials also have an implicit curriculum that they hope to convey to students. However, in order for this implicit curriculum to be conveyed, instructors need to be aware of these intentions. In this paper, we explore this awareness as an issue of a match or mismatch of epistemological framing of instructors and curriculum designers. We know that when instructors consider taking up a new curriculum, they act effectively as learners, and thus, their epistemological framing of the learning environment influences how they implement instructional practices. The epistemological resources that are activated when faculty consider their classroom influences the choice of curricula as well as the implementation and adaptation of those curricula. This can lead to benefits or challenges. For example, faculty who maintain a transmissionist view of learning may view clickers as a means to test students' declarative knowledge or to take attendance. On the other hand, the use of clickers may provide faculty an opportunity to carry out formative assessment and ultimately expand the set of epistemological resources that the faculty member draws upon $[41,42]$.

In this paper, we examine existing UMI curriculum designs in relation to the implicit curriculum [38]. We focus specifically on the epistemological issues about the nature of scientific knowing and learning that are built into our curricular materials. Our focus on the instructor creates a question of how curriculum designers communicate the implicit curriculum to potential users of curricular materials. Specifically, we are interested in articulating the set of epistemological resources the curriculum designers expect to be activated by the curriculum when instituted in the classroom. For this paper, we will use "beliefs" to refer to 
the stable set of epistemological resources the designers of curricular materials intend to communicate as the implicit curriculum. We will refer to the curriculum as the artifacts of these beliefs and ask in what ways the artifacts cue the intended set of epistemological resources to a prospective instructor. In an ideal scenario, there would be near perfect alignment between the beliefs communicated by the designers of the curriculum and the epistemological resources activated by the prospective instructor.

\section{B. Artifacts of designer beliefs are embedded in curricular materials}

With UMI, there is an established set of curricular materials, instructor guides, and student activities that support implementing the curriculum with the intention that other instructors may want access to the materials [43]. Despite their easy access online and promotion through workshops, the materials have not been widely adopted and have been used primarily by developers of the instructional materials. One reason for this is the time, effort, and infrastructure constraints of those looking to implement UMI. We also believe that there are assumptions made by the designers about the nature of knowing and learning that are not articulated in the curriculum, and thus make it hard for instructors to consider taking up.

In the case of UMI, we will investigate the curriculum's communication of three foundational beliefs (listed below). However, we do not mean to say that these are the only beliefs built into the curriculum. For example, the broad set of epistemological beliefs about learning from the designers of UMI have already been thoroughly described in the Modeling Theory of Science and Modeling Theory of Instruction $[17,21,23,44]$. We choose to focus on these three beliefs in this paper because they are particularly relevant to the management of the board meeting, which we have found to be a challenging element for even experienced instructors to implement.

1. Scientific knowledge is built of a set of shared conceptual models. - These conceptual models are not held by an individual, but are distributed across the class [20]. These models are developed through person-person interactions and appropriated by individuals, so that each person has a unique version of the shared conceptual model.

2. Conceptual models are composed of coherent, coordinated representations. - The process of building, validating, deploying, and ultimately revising these coordinated sets of representations is known as modeling, and is the process by which knowledge is built.

3. Students have agency to construct (with guidance) knowledge that aligns with standard accepted understanding of physics, and that this guided epistemological agency [45] allows students to take ownership of the knowledge and knowledge generation processes.

Opportunities are built into the class for the development of shared conceptual models distributed across a classroom. Students are expected to solve and present to one another different problems as a means to give the class exposure to multiple problems. Frequently in class, students will perform different experiments, or will design their own experiments. This naturally leads to different experiences. Additionally, one of the means of guiding students is that during small group interactions, instructors "seed" ideas to some groups and different ideas to others [31]. The expectation is that these groups then bring these ideas to the board meeting. This allows for the conceptual model to develop in shared ways across the classroom community [21].

Second, University Modeling Instruction relies heavily on varied representations, not just on equations, as models are built up of sets of coherent representations [28,29]. The UMI curriculum materials rely heavily on instructors viewing understanding and representing physics as involving more than equations alone. Since representations are built into the curriculum, we expect that representations are incorporated as an essential part of problem solutions. Building skill with creating and interpreting representations is part of physics, and thus should be assessed. As a result, we see the view of representations as an essential part of understanding physics as a productive belief to implementing UMI.

Finally, the curriculum materials for UMI are designed to grant students epistemic agency [45]; thus, instructors have to accept a role that is not necessarily at the center of the classroom. Instead, instructors play a guiding role in activities, while prioritizing students' role in developing models. This allows the students to lead discussions, which frequently means discussions do not proceed directly from question to answer. Often in board meetings, this means that wrong answers are expressed. These wrong answers can be productive as they promote further discussion, with students challenging and reasoning through the answers. However, guiding this discussion requires a willingness from the instructor to be flexible and to allow a discussion to flow in directions that are not predetermined. It is productive to have instructors that view students as capable of leading their learning (with guidance). As a result, the curriculum materials are not developed as a script to be followed and the discussion for an activity is not entirely predictable, which can be unsettling for instructors who have not cued a resource of student epistemological agency.

Some elements of the epistemological beliefs of the developers are evident in the UMI curricular materials, but in many cases there are ways of interpreting the artifacts of these beliefs in ways that are not aligned with the designers' intentions. In the subsequent sections, we will (1) outline our methods and introduce the student-facing and 
instructor-facing materials that have been developed for the UMI curriculum, particularly focusing on one activity from the circuits unit of introductory electricity and magnetism (Sec. III), (2) demonstrate how the epistemological beliefs of the developers are evidenced in the materials (Sec. IV), and (3) highlight what is missing from the materials in order to run the circuits activity as intended (Sec. V).

\section{METHODS AND CONTEXT}

In this section, we outline the approach, methods, and context for the subsequent analysis of the three epistemic beliefs in the UMI curricular materials. First, we position ourselves as the authors of this paper, then outline our approach to the analysis of a single activity from the UMI electricity and magnetism (UMI-EM) curriculum. We then position the activity from the UMI-EM curriculum within the context of the course structures, place the activity in the context of the curriculum sequence, and describe the activity and curriculum materials that we use in our analysis.

\section{A. Positioning of the authors}

As the authors of this paper, it is important for us to position our experience and interaction with the UMI curriculum. First and foremost, we all have significant experience in developing and teaching the UMI curriculum. Brewe (second author) developed the student-facing activities of the UMI mechanics (UMI-Mech) curriculum over the past decade, with the implementation and instantiation of the MTS and MTI articulated previously [23]. Sawtelle (last author) was on a curriculum development team as UMI-Mech was preparing to launch a website with materials. As a new instructor to UMI, she initially developed the instructor guides used with the UMI-Mech curriculum, which have become pivotal to communicating the intent of the activities to other instructors. Monsalve (third author) has extensive teaching experience of the UMI curricula, assisted in revisions of the curricular materials for UMIMech, and has led training of new instructors in UMI. McPadden (first author) developed the UMI-EM curriculum, including both the student-facing activities and the instructor guides [46]. Since their work in UMI-Mech and UMI-EM, Brewe, Sawtelle, and McPadden have adapted and built on the UMI curricula in different contexts, including physics for life science majors, a hybrid problem-based learning and studio context for science and engineering majors, and a lecture context without laboratory equipment.

Thus, we are coming to this paper from the perspective of the curriculum designers. When we talk about the "epistemic beliefs of UMI," we are articulating what we see as the beliefs that we have attempted to build into the UMI materials. This paper was borne out of the conversations and reflections on our own materials, particularly as we each tried to apply the same UMI framework to a different context. This process forced us to articulate our own assumptions and beliefs as curriculum designers. We found these to be subtle and often implicitly built into our materials. In this paper, we attempt to highlight how we see three of these beliefs manifest in the curriculum materials.

\section{B. Approach and analysis}

This paper was intended as a theoretical discussion of the epistemological beliefs built into UMI by curriculum designers. As such, we collectively identified which beliefs to focus this paper around and picked an activity from the UMI curriculum to illustrate how these beliefs were embedded in the curricular materials. As such, we use the curricular materials as our "data source" for the analysis. We use the phrasing and structure built into the curricular materials as the evidence of the epistemic beliefs that were built in by the designers. We then offer different ways that we could see these materials interpreted by instructors, highlighting both the interpretation that was intended by the curriculum designers and possible interpretations that could work against the intended design. These possible interpretations are offered as hypothetical statements that we could envision other instructors making. They are not intended as anecdotes and do not come from recorded observational data. We see this paper as the first in a larger project, which would include interviews with instructors who did not participate in the curriculum design about their beliefs and how those align with the curriculum materials.

In order to decide which epistemological beliefs to include in this paper, we started by having four conversations spread out over six months about how we share curricular materials with new instructors and what assumptions we make about how instructors interpret those materials. From these conversations, we created a list of what we thought was missing from the materials that we created. From this list, we decided to focus on the beliefs that had a strong impact on the board meeting discussions for the following two reasons: (a) there are no studentfacing materials for the board meeting, which leaves the shape of the discussion largely up to the instructor's interpretation, and (b) while the board meetings are critical course structures in UMI, there has been little discussion of how they function in the literature. We then also narrowed to the beliefs that could be interpreted in multiple ways by instructors and that we could see manifested in the curriculum materials (rather than just a belief about practice). While we recognize that there are many other epistemological beliefs held by the designers that are likely present as artifacts in the curricula, this process led us to the three beliefs presented in the analysis below: (1) learning is distributed across the classroom, (2) multiple representations are central to students' construction and 
understanding of the models, and (3) students' epistemic agency is emphasized in the learning process.

After identifying the beliefs, we decided to focus our analysis and discussion around a single activity from the UMI-EM curriculum. Ultimately, we decided on the Combining Resistors activity for several reasons. First, it occurs early in the modeling cycle, thus requiring less context as its focus is to generate the rules for Combining Resistors in a circuit. Second, the activity at first glance seems rather simple (only $1 / 3$ of a page on instructions and space to draw circuits), while the instructor guide indicates that this should be a long activity (90 minutes is budgeted for part 1 alone) to build toward the discussion points for the activity. The discrepancy between the student-facing materials and the instructor guide highlights an interesting place in the curriculum, where the developers are making assumptions about how to move from the student-facing activity into the larger board meeting. Third, this activity provided concrete examples of the three beliefs that we identified.

\section{Positioning of the activity of analysis}

In this section, we provide the context, positioning, and description of the Combining Resistor activity within the UMI-EM curriculum. First, we describe the general UMI curricular structures, which are included in the Combining Resistors activity. Next, we describe where in the unit the Combining Resistors activity falls and what students would have learned in the previous activity. Then, we provide a brief overview of a relatively new representation called Power Boxes, which was designed for and built into the UMI-EM curriculum. This representation is featured heavily in the Combining Resistors activity, and thus, it is worth providing some context on the representation. Finally, we describe both the student-facing and instructor-facing documents for the Combining Resistors activity that we used as the unit of analysis.

\section{UMI curricular structures}

The UMI curricular materials are currently organized by week throughout the semester (e.g., 15 weeks of course outline), consisting of a set of activities and homework (student-facing documents) for that week with an associated instructor guide (instructor-facing documents); in some cases, video vignettes accompany the instructor guide. These are the materials that are available on the UMI website, which would be shared with an instructor interested in using the UMI curriculum.

The format of each activity varies, depending on where in the modeling cycle the activity falls. There are three general forms of activities: investigation activities, coordination activities, and application activities. An investigation activity will typically occur at the beginning of the modeling cycle, with the purpose being to explore a phenomenon (either theoretically or empirically), to generate questions about that phenomenon, and to produce a set of rules from the initial investigation about how that phenomenon works. A coordination activity typically follows the investigation activity (or activities). Coordination activities are designed to focus students on connecting the new concepts and rules from the investigation to their previous knowledge and to the various representations that are used to describe that phenomenon. The investigation and coordination activities serve as the "model building" activities and the place where new knowledge is generated and abstracted by the class. The application activities are then designed so that students have the opportunity to take the model that they built (over the previous activities or class periods) and apply it to realworld contexts. In these activities, students are asked to create a coordinated set of representations for the phenomenon (e.g., system schema, energy pie charts, word description, force diagram, etc.) in addition to solving for any missing quantities [29]. This holistic approach to problem solving is highly emphasized over problems that ask students to solve for one particular quantity [23].

The current version of the instructor guide for UMI is a weekly guideline for time management of six studentcontact hours along with the purpose of the activities, student generated whiteboards, and board meetings. Each week has a breakdown of the activities, the corresponding white board questions, the important points that should be discussed in the board meeting, and recommended homework for the students. The instructor guide states the purpose of each activity including the content knowledge needed or generated. Occasionally, the instructor guide indicates that an idea should be "seeded." The "seeding" tells an instructor that a representation or a formula requires an introduction from an instructor (be it a faculty member, teaching assistant or learning assistant) to formalize the idea during small group discussion [31]. The instructor guides for the activity can also have logistical notes for the instructor to manage small group collaboration or to split the activity into parts during class. The guidelines for the student whiteboards typically have the aforementioned three questions for investigation activities (what did you learn, what patterns or rules do you see, what do you have questions about), a suggested prompt for explanation in the coordination activities, or suggested phenomenon for the application activities. The guidelines for the board meeting have the bullet points of the concept and/or connection of the material that students could cover.

\section{Situating the activity in the curriculum}

For context, the Combining Resistors activity occurs in the seventh week of class, following five weeks focused on electrostatics and one week on introductory circuits. It is the second investigation activity in the modeling cycle on circuits, following an investigation around voltage and current for a single light bulb and for a single resistor. In the 
first part of the previous activity, the students would have come up with rules for basic circuits, such as current will only flow around a closed path, the current is the same everywhere in the circuit (resistors do not "use up" current), there is very little change in voltage over the wires in the circuit, the change in voltage across the battery is the same as the change in voltage across the resistor, and the energy from the battery is transferred to internal energy (heat) at the resistor. This last rule opens up a discussion about energy versus power in circuits and facilitates the introduction of a new representational tool called Power Boxes [47]. We recognize that this is a new representation that is uncommon to most physics course, so we briefly describe the Power Boxes in the following section. In the second part of the activity, students then build quantitatively on these rules to develop Ohm's law. In summary, going into the Combining Resistors activity, students have already defined and measured current, voltage difference, resistance, and power; however, up until this point, students have only been working with a single circuit element (either a light bulb or resistor) that is connected directly to a battery or power supply.

\section{Power Boxes}

Power Boxes were introduced to the UMI-EM curriculum in 2016 and published by McPadden et al. in 2018 [47]. We briefly summarize this representation here as it is a relatively new representation to the physics education community. Power Boxes were introduced in the UMI-EM curriculum as a means to represent the energy in a circuit. More common energy representations, such as energy pie charts, energy bar charts, energy tracking diagrams, etc., often fail when applied to circuits contexts due to a lack of ability to track energy around multiple paths or to address the energy of more than one electron [47]. Rather than focusing on energy, Power Boxes were designed to help students focus on power around a circuit by visually representing changes in potential and current around a circuit.

There are two pieces to Power Boxes-the tracking Power Boxes and the summary Power Box. The vertical side of each box represents the potential available in the circuit, whereas the horizontal side of the box represents the current. Thus, within the box, the area represents the power available in the circuit $(P=I V)$. Tracking Power Boxes are used to help students track changes in potential and/or current at different locations around a circuit, which can then be used to construct a summary Power Box of the whole circuit.

As an example of Power Boxes in use, consider the circuit shown in Fig. 1, where $R_{1}=10 \Omega, R_{2}=5 \Omega$, and $R_{3}=3 \Omega$. The tracking Power Boxes are shown on the right in Fig. 1(a). Starting with point A, the entire box is shaded in black, which indicates that there is the full amount of potential and current from the battery at this location. At location $\mathrm{B}$, after passing $R_{1}$, there is a drop in potential (indicated by a decrease in the vertical black shading), though this does not change the current (indicated by the full horizontal width being shaded). The blue box represents then the change in potential at $R_{1}$. At points $\mathrm{C}$ and $\mathrm{D}$, there is no drop in potential as there is no circuit element between $\mathrm{B}$ and $\mathrm{C}$ or between $\mathrm{B}$ and $\mathrm{D}$, but there is a junction. This would result in a division of the current (which is represented by the splitting of the black shading on the horizontal axis into "bins") such that $R_{2}$ has less (a) Tracking Power Boxes

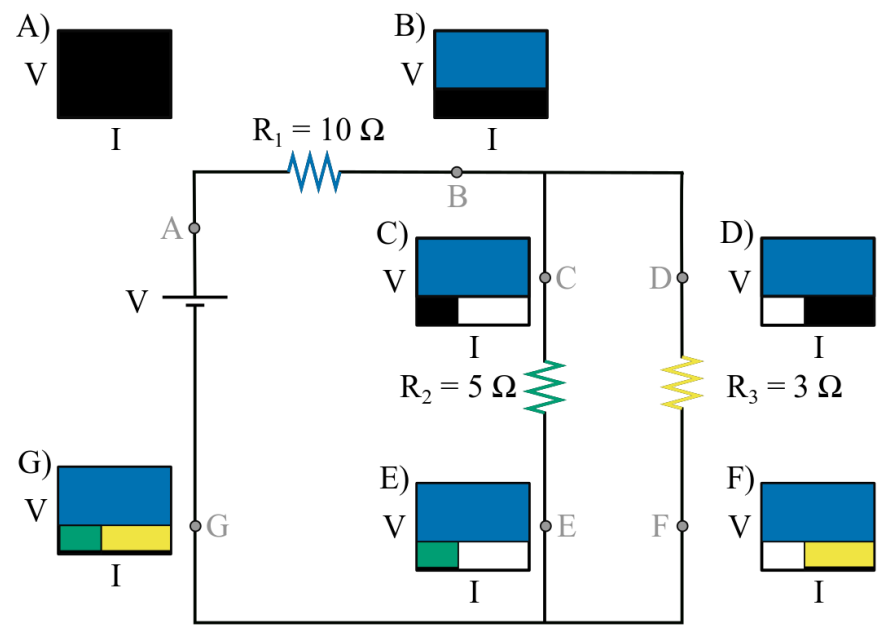

(b) Summary Power Box

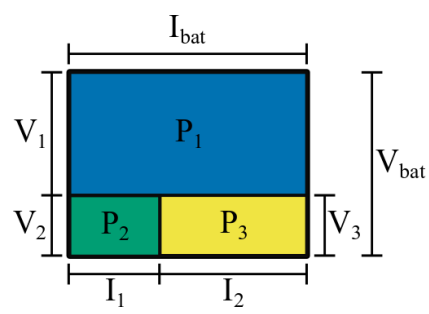

FIG. 1. Example of the (a) tracking Power Boxes and (b) summary Power Box used for a simple direct current (dc) circuit. The Power Box representation shows the changes in potential difference and current around a circuit as a tool to think about power in the circuit. 
current than $R_{3}$. At point $\mathrm{E}$ (and similarly at point $\mathrm{F}$ ), there is a final decrease in potential, indicating the potential drop across $R_{2}$ (or $R_{3}$, respectively); however, there is still current at this location, so this is indicated by a thick black line at the bottom of the Power Box. Finally at point $\mathrm{G}$, the line at the bottom indicates that there is the full amount of current present at that location with zero potential.

The summary Power Box [shown on the left in Fig. 1(b)] then summarizes these changes in potential and current for the whole circuit. We have color coded these sections for clarity (though that may not be expected of students).
From the summary Power Box, it becomes easy to judge where energy is being used at a faster rate in the circuit based on the relative area sizes. Power Boxes can then also be used to help students construct Kirchoff's node and loop rules. For example, it is clear from the diagram that $V_{1}+V_{2}$ must equal the total voltage from the battery $\left(V_{\text {bat }}\right)$. For further examples or details on the Power Boxes, see Ref. [47].

\section{Description of curricular materials for the activity}

The student-facing portion of the Combining Resistors activity is shown in Fig. 2 [48]. In the first part of the

\title{
Activity - Combining Resistors
}

Today we are going to start exploring more complicated circuits with multiple resistors. In this activity you will have access to:
- Power Supply
- 8 resistors
- Wires
- Multimeter and probes

\begin{abstract}
Part 1 - Single Loop
To start, we are going to investigate circuits where all the elements are in a single loop. Your job is to create SIX different circuits - using a maximum of THREE resistors in each circuit. Set the power supply to $5 \mathrm{~V}$ and using the multimeter, take measurements of the voltage across and current through each resistor.

You will be whiteboarding what you learned from the 6 circuits: What rules can you make about the voltage? What rules can you make about the current? What rules can you make about the resistance? What else did you learn?
\end{abstract}

For each of your circuits, draw the circuit diagram, the power boxes, AND record your measurements.

Circuit \#1:

Circuit \#4:

Circuit \#2:

Circuit \#5:

Circuit \#3:

Circuit \#6:

Part 2 - Multiple Loops

Now, we are going to investigate circuits where each resistor is in its own loop. Again, your job is to create SIX different circuits - using a maximum of THREE resistors in each circuit. Using the multimeter, take measurements about the voltage across and the current through each resistor.

You will be whiteboarding what you learned from the 6 circuits: What rules can you make about the voltage? What rules can you make about the current? What rules can you make about the resistance? What else did you learn?

For each of your circuits, draw the circuit diagram, the power boxes, AND record your measurements

Circuit \#1:

Circuit \#4:

Circuit \#2:

Circuit \#5:

Circuit \#3:

Circuit \#6:

Page $1 / 1$

FIG. 2. Student-facing document for the Combining Resistors activity in the UMI-EM curriculum. Note that the spacing has been altered from the original version to better fit the page. 
activity, students are asked to create six different circuits with the restrictions that (1) all the circuit elements must be in a single loop (limiting to the case of series circuits) and (2) they can use a maximum of three resistors in each circuit (however, changing the order of the resistors would count as a different circuit). For each circuit, students are asked to take measurements of the voltage across and the current through each resistor. They are then prompted to think about the rules that they can make for resistors in a single loop. The second part of the activity then broadens to have students investigating resistors that are in separate loops (or are in parallel). They are asked again to create six different circuits and restricted to using three resistors in each circuit. Much like in part 1, they are asked to take voltage and current measurements in each of their circuits and articulate the rules for when resistors are in multiple loops (or in parallel). In each of the parts, students are asked to construct their circuits, record their measurements, draw the circuit diagrams for their circuits, and construct the Power Boxes for their circuits.

The instructor guide for part 1 of this activity is shown in Fig. 3 [49]. (For brevity, we chose to show only part 1 of the corresponding instructor guide as part 2 follows a very similar format for parallel circuits). The instructor guide

In Class Activity Plan

Week 7: Series and Parallel Resistors

Week Notes:

90 min Activity - Combining Resistors - Part 1

PURPOSE - Explore and establish the rules of series circuits.

Teaching Notes:

- Part 1 -we want to restrict students to looking at series circuits, just for simplicity.

- Keep students using only using 3 resistors at a time. This forces student to look at combination with different resistors and combinations with the same resistors.

Whiteboard

PURPOSE - Share experimental results and what was learned about series circuits

- What did you learn?

- What rules can you make?

- What doesn't make sense?

Board Meeting

PURPOSE - Come to consensus on the rules about series circuits.

Important Points:

- Have students explain what circuits they tested and what they measured

- Define "in series" - when two circuit elements have the exact same current they are in series

- So all of the charges that go through one circuit element go through the second element

- Rules of Series Circuits

- The current is the same everywhere in the circuit - This should make sense, not losing charges

- The voltage drops across each resistor

- The voltage across each resistor adds up to the total voltage from the power supply.

- This is sometimes called the Loop Rule or Kirchoff's Loop Rule

- The voltage drop across a wire is very small

- Most of the time we will make the assumption that wires are perfect meaning the voltage does not drop at all on the wire.

- Voltage drop is proportional to resistance

$\circ$ Bigger resistors have bigger voltage drop

- Equal resistors have the same voltage drop

- Power boxes for elements in series

- Current stays the same so horizontal should always be the same

- Voltage would drop across each resistor

- By the time that you return to the battery, only left with current line.

- Total power box for the circuit is divided horizontally - as the current stays the same, but there is a change in voltage across each resistor.

- Area in total power box tells us about energy use

- largest area $=$ brightest bulb or highest temperature or most energy use

FIG. 3. The instructor-facing document for the Combining Resistors activity in the UMI-EM curriculum. This is the first of two pages for the activity. Note that the spacing has been altered from the original version to better fit the page and part 2 was omitted because of similarity to part 1. 
gives an estimate of approximately 90 minutes for the first part of the activity. At the top of the guide, there are teaching notes that briefly explain the reason for the restrictions and the purpose of the activity. The teaching notes are then followed by the whiteboard questions that students should answer and a bulleted list of the important points that should be covered in the board meeting. While the list is bulleted, the order of the points is not meant to be restrictive and could occur in a different order depending on the flow of conversation in the larger discussion. For the first part of the activity, the "important points" in the discussion include defining what it means to be "in series," articulating the rules for current and voltage difference when resistors are connected in series, and articulating how those rules then relate to Power Boxes as a representational tool for analyzing circuits.

\section{ARTIFACTS OF EPISTEMOLOGICAL BELIEFS IN THE MATERIALS}

Within both the student activities and the instructor guide described above, there are artifacts of curriculum designer's epistemological beliefs left as curricular elements. As we stated before, there are likely many beliefs built into the materials; however, we will focus our discussion on three of those particularly relevant for being able to conduct the board meeting following the activity: (1) learning is distributed across the classroom, (2) multiple representations are central to students' construction and understanding of the models, and (3) students' epistemic agency is emphasized in the learning process. The artifacts of each of these epistemological beliefs is described below in the context of the Combining Resistors activity shown in Figs. 2 and 3.

\section{A. Learning is distributed across the classroom}

The student-facing materials communicate the belief about learning being distributed across the classroom in the wording and scaffolds of the activity. In part 1 of the activity, students are asked to create six different circuits and prompted to take measurements of the voltage and current through each resistor. The phrasing of this investigation is intentionally open ended. Students are given constraints to work with (i.e., a maximum of three resistors, the circuit elements should be in a single loop), but beyond that the choice and design of the circuits is up to the students themselves. For example, they could pick circuits with resistors of the same resistance, with a varying number of resistors, resistors of varying resistance, vary the order of the resistors, etc. In giving an open-ended prompt, this means that each group of students is creating a different set of six circuits.

Similarly, the white board prompts in the activity are intentionally open-ended. Students are simply asked to think about the rules that they can make for voltage, current, and resistance, along with anything else that they learned in investigating their circuits. Instead of a direct prompting about a particular rule, this open-ended prompt means that students will come to the discussion with a wide variety of rules, based on what they found from the six particular circuits that they tested. By keeping the prompts open ended, students are able to come up with the rules they are finding that make sense based on their circuits and their measurements.

Since each group is only creating six circuits, it is not expected that every group has all of the voltage rules or all of the current rules in the board meeting. Rather, students are expected to be able to articulate the rules they found from their specific circuits and measurements. When the groups then come together for the board meeting, there is a wide variety of circuits tested and rules that each group found.

Within the instructor guide, this designer belief is evident in the purpose of the whiteboard time and the board meeting purpose, which focus on "sharing experimental results" and "coming to consensus on rules." Since students are intended to investigate different circuits, it is important that they have the opportunity to share what they did since it is likely different from what their neighboring group did. Likewise, there will be negotiation and consensus needed between the groups to develop the rules for series circuits. For example, if one group tested primarily circuits with resistors of the same resistance, they may come up with a rule that says the voltage across each resistor is the same. Whereas, if another group tested resistors of different values, they may have a rule that a larger resistance means a larger potential difference. The board meeting allows the space for students to share these findings and what they did in order to come to consensus on the rules for series circuits. This is further evidenced by the first bullet of the board meeting, which emphasizes that students should explain what they tested and what they measured in investigating their circuits.

Thus, the structures of this activity and the following discussion are based on the idea that the student groups have all tested different circuits. This provides a wider variety of circuits and a wider variety of rules, which can then be negotiated and formalized in the board meeting.

\section{B. Multiple representations are central to students' construction and understanding of the models}

The student-facing materials demonstrate the belief of valuing multiple representations in two sections. First, students are asked for each circuit to have a circuit diagram, Power Boxes, and a table of measurements made on the circuits (i.e., a table of relevant current and voltage measurements). Second, students are given the whiteboard questions that appear in the instructor guide but are contextualized specifically for the understanding single loop circuits. For example, "what rules can you make about 
voltage?" The open-ended nature of the question to ask for a rule allows students to answer with multiple representations that are appropriate to explain the phenomena of single loop circuits.

In the instructor guide, the designers communicate the epistemological belief of multiple representations being central to model construction as dispersed bullet points through the important points of the board meeting. Explicitly, the instructor guide highlights both Kirchhoff's rules and Power Boxes as resulting from this investigation. The board meeting is the place where students' measurements in the circuit (the first bullet) are expected to coordinate with both of these formalized representations. Power Boxes are still a new representation at this stage in the course, and thus, this activity outlines specific elements of that representation.

\section{Guided epistemic agency is emphasized in the learning process}

Within the curricular materials, there is a balance between student agency and the guidance that they are given. It is important to the classroom that both parts of this epistemological belief are activated. We have already discussed how the student-facing materials in the Combining Resistors activity are phrased so that students are free to choose and design their own circuits. This phrasing emphasizes the belief that learning is distributed across that classroom, but also cues resources about providing students with autonomy in the learning process. Not only do they have to design the circuits, they have to make decisions about which resistors they want to use (how many, what values of resistance, the same resistance or different resistance). They also have to make decisions about where they place their multimeters to measure either voltage or current. This provides students with ownership over their circuit design and experimental decisions.

In the instructor guide, students' epistemic agency is communicated through the structure of the board meeting. For example, the first bullet point has students explaining what circuits they tested, what they measured, and what they found. Thus, students should be leading this discussion as opposed to the instructor highlighting student results. With the students leading this discussion, the discussion points are not anticipated to happen in any particular order. This is reflected in the choice of bullets for the discussion points as opposed to a numbered or ordered list. Furthermore, the amount of time budgeted for this activity and discussion is 90 minutes, shown in the top right corner of the instructor guide. This is a reflection that despite the simplicity of the activity, there is a lot of negotiation in the student-led discussion. Because student epistemic agency and ownership is emphasized (especially in the discussion), this means that the discussion will likely not be efficient in terms of time and will require students to work out ideas with one another.
Within the student-facing materials, the "guided" part of this designer belief is communicated through the limitations or constraints that students are asked to work in. For example, students are asked to "investigate circuits where all the elements are in a single loop." This constrains students to focusing on only series circuits; thus, the rules that students are asked to come up with will be focused on only series circuits. The other constraint that helps to guide students in this investigation is that they can only use "a maximum of three resistors in each circuit." This prevents students from just adding one more resistor to make a new circuit. By adding this constraint, the designers are communicating a balance of student agency and guided direction by asking the students to be creative in their circuit design and to consider combinations of resistors that they may not have before (i.e., two resistors with the same resistance with one different). Within the instructor guide, the "guidance" shows up as teaching notes to the instructors. In these teaching notes, the developers explain why there are limitations placed in the student-facing materials, albeit they are short notes and require some interpretation on behalf of the instructor.

\section{IMPLICIT CURRICULUM IN INSTRUCTOR GUIDE AND STUDENT MATERIALS}

In the previous section, we highlighted how the epistemological beliefs of the curriculum designers were communicated in the curricular materials of the Combining Resistors activity. However, the instructor also activates their own epistemological resources when teaching the course and using the materials. Since there are no explicit directions particularly in how one translates between the student-facing activity and the guidelines provided in the instructor guide, the set of epistemological resources activated by the instructor is crucial to the interpretation of the instructor guides and materials. In this section, we articulate how the designers' beliefs outlined above are implicitly built into the curriculum and how those beliefs may interact with the beliefs held by instructors.

\section{A. Learning is distributed across the classroom}

Despite the activity and instructor guide incorporating distributed learning into their structure, the materials do not communicate how the instructor is supposed to use these materials. In the design of the activity, it is not intended that every group come up with all the rules about series circuits. Rather, they are intended to come up with the rules that are related to the circuits they designed and the measurements they took. This means that prior to the board meeting, the instructors are walking around to see what each group is testing. As they go, the instructors should be helping the students formalize the rules that make sense to their group's experiments. If none of the groups are testing a particular rule, the instructor might suggest to one or two groups that they design a circuit in a particular way. This suggestion 
might be to a group who finishes early and has some time before the board meeting, or it might be to a group who is struggling to come up with a fifth or sixth circuit to test. In this way, the instructor can emphasize the distributed nature of learning when planning and supporting the board meeting that follows. However, within the given materials themselves, there are no directions for what the instructor should be doing during the time that students are working on the activity. It is assumed that the instructors know what to do with this time, rather than these sorts of directives being communicated through the curriculum materials.

Additionally, this means that when the instructor calls the board meeting, each group is expected to come with a different set of rules. It is not expected that any one group has a complete set of the rules, but rather that the rules listed in the instructor guide are distributed among the groups. Over the time period of the discussion, a complete set of the rules is expected to be negotiated and pieced together using the information collected by multiple groups. Again, this is not stated in the curricular materials. Instead, the "important points" listed in the board meeting are the complete set of rules that should be pieced together by the end of the board meeting. Even more importantly, there is nothing in the materials that points to how the instructor moves from the rules generated in pieces by the students to the main points presented in the instructor guide. The instructor guide does not explicitly state that learning is distributed or how instructors should interpret the board meeting notes through that lens.

Without specifics for the instructor in the curricular materials, it is quite possible that the instructor does not activated the "learning is distributed" set of epistemological resources. As a result, the instructor may change the purpose or design of the activity unintentionally. For example, they may expect that each group come up with "all" the rules from the activity. This may mean that the instructor modifies the activity so that students are given the "right" circuits to test that will lead to all the rules, or they may ask students to build and test more circuits so they can come up with the "complete" set on their own. In this design, the activity may take up much more class time than anticipated since the work being asked of the students is much higher. This may also lead to a pointless board meeting since each group would then come in with the same set of rules. This would leave nothing for the students to negotiate, discuss, or come to consensus about. Depending on the goals of the instructor, this may unintentionally undermine the purpose of the activity and/or the following board meeting.

\section{B. Multiple representations are central to students' construction and understanding of the models}

While many designers of transformed pedagogies assume that multiple representations are productive, this belief about the nature of knowledge is core to UMI's construction of models. In the UMI classroom, students learn that models are a coordinated set of representations. Thus, the epistemological belief of multiple representations being central to learning is key to interpreting and implementing both the instructor guide and the student-facing materials. The student materials prompt students in this direction-asking them to record their circuits in multiple forms (e.g., make a table of measurements and draw a circuit diagram) and to draw Power Boxes. However, students are apt to skip the representations they find unnecessary, and as a result, a key activity of an instructor of UMI is to focus attention on the coordinated set of representations. As the instructor is moving around the room, they are looking for representations to work together to tell a consistent story - or to develop a key rule. Working hand in hand with the belief of distributed learning, the UMI instructor looks for groups that have representations that are consistent within their data collection, but where the representations highlight differences across groups.

Not found in the instructor guide are any notes about how to pull the different group representations together to formulate a rule in the board meeting. Without activating a set of epistemological resources around multiple representations being central to learning, we might see instructors using a single group to write Kirchhoff's loop rule in equation form without noting how it is represented by Power Boxes. Similarly, in the board meeting, we might find instructors wondering what they are meant to do with a group that did not use any equations at all. In this version of the classroom, we might find some students who never really understand circuits or find that they do not have a good tool to help them think through complicated problems. Without a commitment to multiple representations, instructors might bypass individual students and end up marginalizing students who do not understand the one representation that appears to be highly valued.

Similarly, without an activated set of resources that highlight the coordinated nature of multiple representations, an instructor might miss key elements that are stated within the materials. A UMI designer reads the bullet "the voltage across each resistors adds up to the total voltage from the power supply" and recognizes that the bullet should show up on students' whiteboards as (1) a formulaic or mathematical expression, (2) a sentence with words, (3) a Power Box, and (4) a sequence or table of measurements. Depending on where each group is in the activity and what they tested, they may have some or all of these representations, which relates back to the belief of distributed learning. Without the belief that all of these representations are valuable, an instructor might mistakenly value only one and miss the varied set of experiments and representations that should be valued during a board meeting.

\section{Guided epistemic agency is emphasized in the learning process}

Within the student-facing and instructor-facing materials, students are seen as epistemic agents, and their role as 
knowledge generators is promoted in the phrasing of the activity and the constraints placed on students' circuit designs. However, this epistemological belief is particularly influential in the interactions between instructors and students, as well as in the role that the instructor takes both prior to and during the board meeting. Despite its importance, this belief is not articulated well in either the literature or as an artifact in the curricular materials $[14,30,31]$. It is assumed that the instructor has activated a similar set of epistemological resources and interprets the materials through this lens. Furthermore, it assumed that the instructor knows how to balance the guidance they are providing with supporting student autonomy in the learning process.

A primary example of how the curriculum fails to cue this set of resources is that there is nothing built into the instructor guide that says how one moves from the student-facing activity to the bullet points for the discussion listed in the instructor guide. This means that many questions are left up to the interpretation and judgment of the instructors in the room: How much guidance does the instructor provide when working with the small groups on the activity? When should the instructor call a board meeting? How much time should be spent with each group? How does the instructor guide the discussion while maintaining students' ideas? When do you leave questions open ended versus telling students? How does the instructor promote a studentled discussion in the board meeting? How do you know when the board meeting is done and students "have it"?

The epistemological resources that are activated for an instructor will influence how they implement the materials. For example, if the instructor resources about student epistemic agency are activated without any resources emphasizing guided inquiry, the activity may be implemented in a pure discovery-learning style. In this framing, the instructor would not help students during the activity time and allow students to create the circuits and rules on their own. Certainly, there is nothing wrong with this framing; however, it is coming from a a different set of epistemological resources than was intended by the curriculum designers. If students are given complete freedom in the activity without guidance from the instructor, this could mean that one or more of the "important points" in the instructor guide do not come up in the board meeting based on students' experiments. Instead the instructor may be forced to introduce those ideas in the board meeting more often than they would like, which undermines the purpose of the activity and the modeling cycle that are built into the class. Furthermore, if the instructor does not see their role as guiding the groups during the activity time, this could mean the instructor does not feel like they have a role during the activity or are sitting idly by for large parts of the class.

Alternatively, if the instructor activates the guided inquiry set of resources without any resources about student epistemic agency, this could lead the instructor to tell the student groups what they "should" find as opposed to working from students' ideas and measurements first. This could lead to students just waiting for the instructor to tell them what to do or to tell them what the answers are without working through their own ideas. Rather than students taking ownership over their own ideas and the learning process, this promotes the idea that the instructor has all the "right" answers and that if students just wait for the instructor, those answers will be told to them. This can also be problematic in the board meeting, where students may become less willing to share their findings unless they are sure they are "right" or they will simply wait until the instructor provides the answers they were "supposed" to find through the activity. This removes the discussion and evaluation from the board meeting, turning into a lecture from the instructor.

Thus, what an instructor emphasizes and how they balance the guidance and student epistemic agency can change the implementation of the same activity with the same instructor guide. In their current format, the curricular materials do not offer any supports or suggestions for how to navigate this balance or implement it while teaching the activity. It is entirely implied or left up to the discretion of the instructor, which can significantly change the purpose or messaging behind the activity and board meeting discussion.

\section{DISCUSSION AND CONCLUSION}

In the previous sections, we have provided an example from the UMI curriculum that shows how three epistemological beliefs of the UMI designers are left as artifacts into the curricular materials: scientific knowledge is built on a set of distributed set of conceptual models, multiple representations support student understanding, and students have epistemic agency which is guided by instructors. These epistemological beliefs were chosen as the focus of the paper since they have a significant impact on the role of the instructor both during the activity time and the board meeting, which has been left out of the discussion in previous publications on University Modeling Instruction. In addition to highlighting how these epistemological beliefs are left as artifacts in the student- and instructorfacing materials, we discussed how these materials may not cue a similar set of resources for an instructor, thus influencing the implementation of the activities. Within the curricular materials, particularly the instructor guide, the designers assume that the instructor using the guide knows how to interpret and move between the activity and instructor guide in a way that is consistent with the designer beliefs.

Currently, the MI materials do not detail how the instructor moves between the provided student-facing and instructor-facing curricular materials. In part, this is because the materials are designed to be flexible. If students are to have epistemic agency in the learning process, they 
should be free to come up with their own ideas and rules from the activity. This makes it difficult for the designers to describe in detail how the instructor should move from the student idea to the "important bullets" since the students' ideas are intended to be the starting point. While it is possible to outline predictions of what students may come up with, it is not guaranteed that students would come up with the same ideas. Additionally, providing one line of reasoning may unintentionally suggest that there is only one path to get from a students' idea to a given discussion point, when, in reality, the discussion may take a variety of forms with different paths to get to the same result. This format offers flexibility to the instructor to build off of and be responsive to students' ideas.

However, this expects a lot from an instructor. It expects an instructor to be able to use the techniques of responsive teaching [50], to be comfortable leading a discussion that is guided yet student centered, and to be flexible and comfortable in a teaching environment that is difficult to prep for. On top of that, much of what is "missing" from the instructor materials are really a set of teaching practices. For example, how to lead a discussion, how to transition from a small-group activity to a large-group discussion, how much time to spend with each group - these are all practices that are not explicit in the curriculum and techniques that are passed from one instructor to another. While these practices are as much a part of the curriculum as the materials, it is impossible (and undesirable) to write down in a document how each of the practices is used in the variety of scenarios that might come up. Not only is this impractical from a design perspective, but it would make the instructor guide overwhelming to read, difficult to implement, and still may not be understandable just from reading off the paper without the practical application in the classroom.

Thus, we argue that simply passing off the curricular materials (only the student- and instructor-facing documents) is unlikely to be sufficient for a new instructor to adopt a curriculum. This problem is exacerbated when there is a mismatch between the epistemological resources cued for the instructor and the beliefs of the curriculum developers. As we have demonstrated in this paper, there are epistemological beliefs built into any set of curriculum materials. These sets of resources are also presumed to be activated in the interpretation of the materials, which are crucial to the adoption and authentic adaptation of the curriculum. Particularly if the intended resources are not activated by an instructor when looking at the curricular materials, there may not be enough supports for the instructor to successfully implement the new teaching style, no matter the demonstrated benefits provided by the curriculum. Previously, workshops at national meetings have been the primary vehicle to help faculty understand the intended epistemological resources prior to implementing the curriculum. However, work on dissemination suggests that other modes of dissemination are also important and may be useful in developing a coherence between instructor and curriculum developer beliefs without workshops [13].
While we have discussed an example from University Modeling Instruction in this paper, we do not believe that this is unique to the UMI curriculum. Rather, all curriculum developers leave artifacts of their epistemological beliefs in every curriculum. The epistemological resources that are activated by an instructor can significantly impact how the materials are interpreted and implemented. The more disparate the beliefs are between those held by instructors and those intended in the curricula, the more likely it is that instructors will need supports beyond the materials in order to authentically implement them as intended by the designers, or will simply choose not to adopt the materials.

Curriculum dissemination must include more than the transfer of curricular materials-especially for curricula that take on epistemological beliefs that are distinct from typical instructional settings. It is important that curriculum designers are able to identify and articulate these beliefs to those who may take up the curriculum. This paper would also suggest that it is unproductive to separate the pedagogical techniques from the curriculum materials as the design and intention of the materials is in part based on their implementation. From this perspective, by focusing curriculum dissemination on only materials or alternatively on only pedagogical techniques, the interplay between the materials, their design, and their implementation is undervalued. Thus, in disseminating a curriculum, having a space to learn both the classroom practices and the materials would offer the greatest opportunity for successful adoption. This is not a new idea-many workshops at the American Association of Physics Teachers Meetings focus on curriculum implementation of specific curricula. Within modeling instruction, the training models that have been successful at the high school and university levels have been extensive summer workshops and apprenticeship or co-teaching models in the classroom, yet these are expensive and instructor-heavy models of dissemination.

Research has shown that active learning curricula are slow to be adopted despite their demonstrated benefits. We aver that one possible reason for this is a lack of articulation of epistemological beliefs that come with an active-learning curriculum, in combination with the fact that the successful professional development models for new faculty are resource heavy in terms of time, money, and possibly travel. We have used UMI as an example from our own development experience, but in doing so, we do not suggest that UMI has a perfect answer to this problem. On the contrary, we have shown places where the materials in UMI are lacking. However, we would urge both curriculum designers and those who disseminate curricula to think about what supports are needed beyond the materials for other instructors to implement the curriculum. With this paper, we hope to start and continue the conversation within the community about how we can better communicate and support new faculty in adopting and adapting new curricula. 


\section{ACKNOWLEDGMENTS}

We would like to thank the members of the curriculum development team, who worked to create the materials for the University Modeling Instruction curricula. This work was also funded in part by the National Science Foundation Grants No. DGE 1038321 and No. DUE 1140706.
[1] E. Mazur, Peer Instruction: A User's Manual (PrenticeHall, Upper Saddle River, NJ, 1997).

[2] C. H. Crouch and E. Mazur, Peer Instruction: Ten years of experience and results, Am. J. Phys. 69, 970 (2001).

[3] J. M. Wilson, The CUPLE physics studio, Phys. Teach. 32, 518 (1994).

[4] E. E. Etkina, S. Murthy, and X. Zou, Using introductory labs to engage students in experimental design, Am. J. Phys. 74, 979 (2006).

[5] E. Etkina and A. Van Heuvelen, Investigative science learning environment - A science process approach to learning physics, in Research-Based Reform of University Physics, edited by E. F. Redish and P. J. Cooney (American Association of Physics Teachers, College Park, MD, 2007), Reviews in PER Vol. 1, http://www.per-central.org/ document/ServeFile.cfm?ID=4988.

[6] L. C. McDermott, Oersted Medal Lecture 2001: Physics education research-The key to student learning, Am. J. Phys. 69, 1127 (2001).

[7] R. W. Chabay and B. A. Sherwood, Matter and Interactions, 3rd ed., edited by S. Johnson (John Wiley \& Sons, Inc., Hoboken, NJ, 2011).

[8] R. J. Beichner and J. M. Saul, Introduction to the SCALE-UP (student-centered activities for large enrollment undergraduate programs) project, Proceedings of the International School of Physics "Enrico Fermi," Varenna, Italy, http://www.ncsu.edu/per/scaleup.html (accessed 7 June 2005).

[9] R. R. Hake, Interactive-engagement versus traditional methods: A six-thousand-student survey of mechanics test data for introductory physics courses, Am. J. Phys. 66, 64 (1998).

[10] S. Freeman, S. L. Eddy, M. McDonough, M. K. Smith, N. Okoroafor, H. Jordt, and M. Pat Wenderoth, Active learning increases student performance in science, engineering, and mathematics, Proc. Natl. Acad. Sci. U.S.A. 111, 8410 (2014).

[11] C. Henderson and M.H. Dancy, Physics faculty and educational researchers: Divergent expectations as barriers to the diffusion of innovations, Am. J. Phys. 76, 79 (2008).

[12] M. Dancy and C. Henderson, Pedagogical practices and instructional change of physics faculty, Am. J. Phys. 78, 1056 (2010).

[13] M. Dancy, C. Henderson, and C. Turpen, How faculty learn about and implement research-based instructional strategies: The case of Peer Instruction, Phys. Rev. Phys. Educ. Res. 12, 010110 (2016).

[14] E. Brewe, L. Kramer, and G. O'Brien, CLASS shifts in modeling instruction, AIP Conf. Proc. 1064, 75 (2008).
[15] D. Hestenes, Modeling methodology for physics teachers, in Proceedings of the International Conference on Undergraduate Physics Education (AIP, New York, 1996), Vol. 399, pp. 935-958, https://doi.org/10.1063/1.53196.

[16] I. A. Halloun, Modeling Theory in Science Education (Springer, New York, 2004), p. 250.

[17] D. Hestenes, Toward a modeling theory of physics instruction, Am. J. Phys. 55, 440 (1987).

[18] N. J. Nersessian, Maxwell and "the method of physical analogy": Model-based reasoning, generic abstraction, and conceptual change, in Reading Natural Philosophy: Essays in the History and Philosophy of Science and Mathematics, edited by D.B. Malament (Open Court Publishing Company, Peru, 2002), Chap. 6, pp. 129-166.

[19] J. Gouvea and C. Passmore, "Models of" versus "models for": Toward an agent-based conception of modeling in the science classroom, Sci. Educ. 26, 49 (2017).

[20] E. Brewe, J. E. Bartley, M. C. Riedel, V. Sawtelle, T. Salo, E. R. Boeving, E. I. Bravo, R. Odean, A. Nazareth, K. L. Bottenhorn, R. W. Laird, M. T. Sutherland, S. M. Pruden, and A.R. Laird, Toward a neurobiological basis for understanding learning in University Modeling Instruction physics courses, Front. ICT 5, 10 (2018).

[21] E. Brewe and V. Sawtelle, Modeling instruction for university physics: Examining the theory in practice, Eur. J. Phys. 39, 054001 (2018).

[22] D. Hestenes, Modeling methodology for physics teachers, AIP Conf. Proc. 399, 935 (1997).

[23] E. Brewe, Modeling theory applied: Modeling instruction in introductory physics, Am. J. Phys. 76, 1155 (2008).

[24] E. Brewe, V. Sawtelle, L. H. Kramer, G. E. O'Brien, I. Rodriguez, and P. Pamelá, Toward equity through participation in modeling instruction in introductory university physics, Phys. Rev. ST Phys. Educ. Res. 6, 010106 (2010).

[25] E. Brewe, L. Kramer, and G. O'Brien, Modeling instruction: Positive attitudinal shifts in introductory physics measured with CLASS, Phys. Rev. ST Phys. Educ. Res. 5, 013102 (2009).

[26] E. Brewe, A. Traxler, J. de La Garza, and L. H. Kramer, Extending positive CLASS results across multiple instructors and multiple classes of modeling instruction, Phys. Rev. ST Phys. Educ. Res. 9, 020116 (2013).

[27] A. L. Traxler, J. V. Mahadeo, D. McPadden, and E. Brewe, Multiple representations and epistemic games in Introductory Physics exam solutions, in , 2014 PERC Proceedings, Minneapolis, MN, July 30-31, 2014, edited by P. V. Engelhardt, A. D. Churukian, and D. L. Jones, pp. 247-250, https://doi.org/10.1119/perc.2014.pr.058.

[28] D. McPadden and E. Brewe, Network analysis of students' representation use in problem solving, 2015 PERC 
Proceedings College Park, MD, July 29-30, 2015, edited by A. D. Churukian, D. L. Jones, and L. Ding (American Physical Society, New York, 2016), https://doi.org/ 10.1119/perc.2015.pr.050.

[29] D. McPadden and E. Brewe, Impact of the second semester University Modeling Instruction course on students representation choices, Phys. Rev. Phys. Educ. Res. 13, 020129 (2017).

[30] D. M. Desbien, Modeling discourse management compared to other classroom management styles in university physics, Ph.D. Thesis, Arizona State University, 2002.

[31] J. L. Durden, E. Brewe, and L. H. Kramer, "Implicit action": Understanding discourse management in modeling instruction, in 2011 PERC Proceedings, Omaha, NE, August 3-4, 2011, edited by N. S. Rebello, P. V. Engelhardt, and C. Singh [AIP Conf. Proc. 1413, 187 (2012)], https://doi.org/10.1063/1.3680026.

[32] E. Goffman, Forms of Talk (University of Pennsylvania Press, Philadelphia, PA, 1981).

[33] D. Tannen, What's in a frame? Surface evidence for underlying expectations, in Framing in Discourse (Oxford University Press, New York, 1993), Chap. 1.

[34] W. A. Sandoval, Understanding students' practical epistemologies and their influence on learning through inquiry, Sci. Educ. 89, 634 (2005).

[35] D. Hammer, A. Elby, R. E. Scherr, and E. F. Redish, Resources, framing, and transfer BT, in Transfer of Learning from a Modern Multidisciplinary Perspective, edited by J. P. Mestre (Information Age, Greenwich, CT, 2005), pp. 89-120.

[36] D. Hammer and A. Elby, Personal Epistemology, in Personal Epistemology: The Psychology of Beliefs about Knowledge and Learning, edited by B. K. Hofer and P. R. Pintrich (Lawerence Erlbaum Associates, Inc., Mahwah, NJ, 2002), pp. 169-190.

[37] D. Hammer and A. Elby, Tapping epistemological resources for learning physics, J. Learn. Sci. 12, 53 (2009).
[38] E. F. Redish and D. Hammer, Reinventing college physics for biologists: Explicating an epistemological curriculum, Am. J. Phys. 77, 629 (2009).

[39] J. Gouvea, V. Sawtelle, and A. Nair, Epistemological progress in physics and its impact on biology, Phys. Rev. Phys. Educ. Res. 15, 010107 (2019).

[40] J. Watkins and A. Elby, Context dependence of students' views about the role of equations in understanding biology, CBE Life Sci. Educ. 12, 274 (2013).

[41] C. Turpen and N.D. Finkelstein, Not all interactive engagement is the same: Variations in physics professors' implementation of Peer Instruction, Phys. Rev. ST Phys. Educ. Res. 5, 020101 (2009).

[42] A. Olmstead and C. Turpen, Pedagogical sensemaking or "doing school": In well-designed workshop sessions, facilitation makes the difference, Phys. Rev. Phys. Educ. Res. 13, 020123 (2017).

[43] University Modeling Instruction, http://univmodelinginstruction.com/.

[44] I. A. Halloun, Modeling Theory in Science Education, 1st ed. (Springer, Dordrecht, The Neetherlands, 2006).

[45] D. Stroupe, Examining classroom science practice communities: How teachers and students negotiate epistemic agency and learn science-as-practice, Sci. Educ. 98, 487 (2014).

[46] D. McPadden, Examining students' representation choices in University Modeling Instruction, Ph.D. thesis, Florida International University, 2018.

[47] D. McPadden, J. Dowd, and E. Brewe, Power boxes: New representation for analyzing dc circuits, Phys. Teach. 56, 362-366 (2018).

[48] Note that Fig. 2 has been edited slightly to remove white space so that the worksheet fits on one page.

[49] Note that Fig. 3 has been edited slightly to remove white space so that the instructor guide fits on one page.

[50] Responsive Teaching in Science and Mathematics, edited by A. D. Robertson, R. E. Scherr, and D. Hammer (Routledge, New York, 2016). 